www.jmscr.igmpublication.org

Impact Factor 5.84

Index Copernicus Value: 83.27

ISSN (e)-2347-176x ISSN (p) 2455-0450

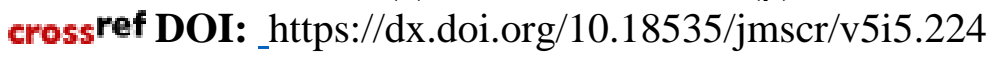

Journal Of Medical Science And Clinical Research

\title{
Grouping of Clinical Isolates of Streptococci by Latex Agglutination in A Tertiary Care Centre
}

\author{
Authors \\ Dr Lancy $\mathbf{J}^{1}$, Dr Anupama ${ }^{2}$, Dr Thara Ann Jose ${ }^{3}$, Dr Lakshmi Balaraman ${ }^{4}$ \\ Department of Microbiology, Govt. Medical College, Thiruvananthapuram, Kerala
}

\begin{abstract}
Latex agglutination technique in an important procedure in clinical laboratories. It is a more rapid and technically less demanding than traditional extraction precipitation methods for the rapid and accurate identification of the Lancefied group of beta haemolytic streptococci. It can be used to detect Group D antigen is streptococci and enterococci as well as group $A, B, C, F$ and $G$ antigens. It is a reliable method for grouping of the beta-haemolytic streptococci and enterococci whenever they are isolated from clinical specimens in culture on steep blood agar in clinical laboratories. A total number of 33 isolates obtained from various clinical specimens received in the 24 hours clinical microbiology laboratory at Govt. Medical College, Hospital, Thiruvananthapuram during a period of 6 months from July 2016 to December 2016 were subjected to Lancefield Grouping by Latex Agglutination test. Majority of the isolates were Group A streptococci 14 (42\%) followed by Group B streptococci 6 (18\%), Group D streptococci and group F streptococci (6\%) and 7 isolates(21\%) were non-groupable.

Lancefield grouping of the beta - haemolytc streptococci by latex Agglutination immediately after their isolation in culture helps the clinician to start treatment at the earliest with appropriate antibiotic without any delay thus providing better patient care in invasive and noninvasive streptococcal infections and to prevent complication.
\end{abstract}

Keywords: Beta-haemolytic streptococci, streptococcus pyogeneslatex agglutination, Lancefied grouping.

\section{INTRODUCTION}

Streptocci are the most frequently encountered gram positive bacteria in the clinical laboratories. Most of the occur as commensals in the oral cavity and skin. Beta-haemolytic streptococci are pathogenic and produces non-invasive and invasive infections in human beings. In the recent years, there has been an increasing inflow of information that haemolytic streptococci are highly prevalent in our areas. They colonise the population producing carrier states and diseases in man. The high incidence of these diseases point to the necessity of adequate laboratory investigations for a proper clinical, bacteriological and epidemiological studies. Conventional Lancefield grouping of beta haemolytic streptococcal isolates is laborious and time consuming. Latex agglutination technique combined with a rapid efficient extraction method allows grouping to be carried out using a single colony from a solid medium.

\section{MATERIALS AND METHODS}

Study design: Descriptive study 
Study population: Patients attending. OPD of Internal medicine with upper respiratory infections patients admitted in medical wards and ICU with suspected streptococcal infections.

Study period: 6 months (July 2016 to Dec 2016)

Study materials: Isolates of streptococci obtained from clinical specimens during the study period.

Age group of patients: 13 years to $80 \mathrm{yrs}$.

Study setting: Department of Microbiology \& Medicine, Govt. Medical College, Thiruvananthapuram

\section{METHODOLOGY}

\section{Collection of samples}

Different types of samples were collected according to the clinical infection from patients under sterile precautions. Exudates from the site of infection were collected by cleaning the site with sterile saline and material collected using sterile double swabs. Pus samples were aspirated using sterile syringe and needle from abscesses after cleaning the surface with sterile saline and surgical spirit. In patients with upper respiratory tract infections, throat swab was collected. Pleural fluid was aspirated under sterile precautions from patients with pleural effusion collected in sterile container. In suspected cases of infective endocarditis. Blood samples were collected by venepuncture under sterile precautions from different sites at half an hour intervals and at least 6 samples were collected. Each sample of blood (5 $\mathrm{ml}$ ) collected and directly inoculated into the blood culture bottle containing $50 \mathrm{ml}$ of Brain heart - Infusion broth and incubated at $37^{\circ} \mathrm{C}$ in an incubator immediately after collection.

\section{Process of the samples}

Exudate (Double swab)

Gram staining was done using one swab to study the morphology, gram reaction and arrangement of the organism present in the clinical specimen Culture was performed using the second swab. The material was inoculated into sheep blood agar plate, Mac conkey agar plate, chocolate agar plate and incubated at $37^{\circ \mathrm{C}}$.
Aspirated pus was inoculated intosheep blood agar plate, Mac Conkey agar plate and glucose broth and incubated aerobically at 370C. One blood agar plat was incubated under anerobic conditions and Robertson's Cooked meat medium was also used to cultivate anerobes.

Blood culture was done by conventional method after overnight incubation at 370C subcultured on sheep blood agar plate and Mac Conkey agar plate.

Identification of bacteria from culture

Sheep blood agar - Beta haemolytic colonies.

Mac Conkey agar - No growth. ]

Glucose broth - Granular turbidity

One colony is picked up from the BA plate with sterile bacteriological loop and gram staining was done after smear preparation. Gram positive cocci arranged in chains will be seen. Catalase test Negative Antibiotic sensitivity of the isolate was performed on Blood agar plate with 0.5 McFarland turbidity suspension and zone of inhibition measured and interpreted according to CLSI guidelines. The antibiotic disks tested were Penicillin (10 units) Ampicillin (10 ug), Cephalexin (10ug), gentamicin Bacitracin (10.04 units), Erythromycin (30 ug) Linezolid (10 mg) and vancomycin (10ug).

\section{Lancefield grouping}

Is a method of grouping catalase negative, coagulase - negative, gram positive Beta haemolytic streptococci based on the carbohydrate antigen found on their cell wall. The system was created by Rebeeca Lancefield, a prominent American Microbiologist. There are 20 Lancefield groups identified named A-V except I and J, based on precipitation reaction with appropriate antisera. Preliminary identification of streptococcal species Group A (streptococcus pyogenes)

Betahaemolysis on sheep blood agar plate.

Bacitracin (0.04units) sensitive

\section{PYR test positive}

Group B - Streptococcus agalactiae

Beta Haemolysis on sheep blood agar

Minute lactose fermenting colonies on Mac Conkey agar 
CAMP test - positive

Hippurate hydrolysis - positive

Group D(Enterococcus faccalis)

Beta - haemolysis on sheep blood agar

Tiny deep pink colonies on Mac Conkey agar.

Growth in $6.5 \% \mathrm{NaCl}$.

Heat resistance test +ve(at 600C / $30 \mathrm{mts})$

PYR test - positive

Aesculin hydrolysis - positive

Group F - Streptococcus anginosus

Beta-haemolysis on sheep blood agar

Produces minute colonies only on BA.

No growth on Mac Conkey agar.

\section{Lancefield grouping of beta haemolytic} streptococci by latex agglutination

Streptococci possess specific polysaccharide cell wall antigens which allow the bacteria to be classified into groups. The antigen is extracted into solution by the action of an enzyme. Solubilised antigen is then added to a suspension of latex particles coated with specially purified rabbit antibodies to the group antigens. If a group antigen is present, that group of latex will agglutinate. If no group antigen is present, the latex stays in smooth suspension.

Latex agglutination test is used as an in-vitro diagnostic test for the identification of streptococci isolated in cultures from clinical specimens in the clinical laboratory especially to determine the Lancefied grouping of A,B,C,D,F and G Streptococci.

\section{RESULTS}

A total no. of 33 clinical isolates obtained from patients admitted with various streptococcal infectious in the surgical wards and the patients attending outpatient department of Medicine of Govt. Medical College Hospital, Thiruvananthapuram were included in the study. Lancefield grouping of the isolates were done by latex diagnostics Ltd. UK. ('Avipath - strep') in the 24 hrsclinical Microbiology Laboratory. agglutination using a kit supplied by Omega

Table 1 : Sample analgesics

\begin{tabular}{|l|c|c|}
\hline Sl. No. & Clinical infection & Total no. \\
\hline 1 & Dental abscess & 5 \\
\hline 2 & Septic arthritis & 4 \\
\hline 3 & Brain abscess & 4 \\
\hline 4 & Infective endocarditis & 3 \\
\hline 5 & Pleural Effusion & 2 \\
\hline 6 & Perianal abscess & 3 \\
\hline 7 & Gluteal abscess & 2 \\
\hline 8 & Scrotal abscess & 1 \\
\hline 9 & Parotic abscess & 1 \\
\hline 10 & Submandibular abscess & 1 \\
\hline 11 & Finger abscess & 1 \\
\hline 12 & Leg ulcer & 2 \\
\hline 13 & Cellulitis & 2 \\
\hline 14 & Hard palate abscess & 1 \\
\hline 15 & Pilonidal abscess & 1 \\
\hline \multicolumn{3}{|c|}{ Total } \\
\hline
\end{tabular}

Table 2 : Specimenwise analysis of isolates (11)

\begin{tabular}{|l|c|c|}
\hline Sl. No. & Nature of specimen & No. $(\%)$ \\
\hline 1 & Exudate (double swab) & $12(36 \%)$ \\
\hline 2 & Throat swab & $2(6.06 \%)$ \\
\hline 3 & Aspirated pus & $10(30 \%)$ \\
\hline 4 & Synovial fluid & $4912 \%)$ \\
\hline 5 & Pleural fluid & $2(6.06 \%)$ \\
\hline 6 & Blood & $3(9.09 \%)$ \\
\hline & Total & 33 \\
\hline
\end{tabular}

Table 3 Age wise distribution of samples

\begin{tabular}{|l|c|}
\hline Age group & No. $(\%)$ \\
\hline $13-20$ & $3(9.09 \%)$ \\
\hline $21-30$ & $5(15.15 \%)$ \\
\hline $31-40$ & $3(9.09 \%)$ \\
\hline $41-50$ & $5(15.15 \%)$ \\
\hline $51-60$ & $7(21.21 \%)$ \\
\hline $61-70$ & $6(18.18 \%)$ \\
\hline $71-80$ & $3(9.09 \%$ \\
\hline $81-90$ & $1(3.03 \%)$ \\
\hline Total & $33(100 \%)$ \\
\hline
\end{tabular}

Table 4 Sex wise distribution of samples received \begin{tabular}{|l|l|l|}
\hline Total No. & Male & Female \\
\hline 33 & $26(78.78 \%)$ & $7(21.21 \%)$ \\
\hline
\end{tabular}

Table 5 Clinical infection according to grouping

\begin{tabular}{|l|c|}
\hline Lance Field group & No of isolates \\
\hline Group A & $14(42.42 \%)$ \\
\hline B & $6(28.12 \%)$ \\
\hline D & $4(12.12 \%)$ \\
\hline F & $2(6.06 \%)$ \\
\hline Non-groupable & $7(21.21 \%)$ \\
\hline
\end{tabular}

Table 6 Group-wise distribution of infections

\begin{tabular}{|l|l|l|l|l|l|l|}
\hline S1. No. & Infections & GAS & GBS & GDS & GFS & NG \\
\hline 1 & Dental Abscess & 2 & 0 & 0 & 0 & 3 \\
\hline 2 & Septic arthritis & 2 & 0 & 0 & 0 & 2 \\
\hline 3 & Infection endocarditits & 2 & 0 & 1 & 0 & 0 \\
\hline 4 & Pleural effusion & 2 & 0 & 0 & 0 & 0 \\
\hline 5 & Cellulitis & 2 & 0 & 0 & 0 & 0 \\
\hline 6 & Parotid abscess & 1 & 0 & 0 & 0 & 0 \\
\hline 7 & Submandibular abscess & 1 & 0 & 0 & 0 & 0 \\
\hline 8 & Hard palate abscess & 0 & 0 & 0 & 1 & 0 \\
\hline 9 & Finger abscess & 0 & 0 & 0 & 1 & 0 \\
\hline 10 & Pilonidal abscess & 0 & 1 & 0 & 0 & 0 \\
\hline 11 & Brain abscess & 2 & 0 & 0 & 0 & 2 \\
\hline 12 & Leg ulcer & 0 & 2 & 0 & 0 & 0 \\
\hline 13 & Perianal abscess & 0 & 2 & 1 & 0 & 0 \\
\hline 14 & Gluteal abscess & 0 & 1 & 1 & 0 & 0 \\
\hline 15 & Scrotal abscess & 0 & 0 & 1 & 0 & 0 \\
\hline & Total & 14 & 6 & 4 & 2 & 7 \\
\hline
\end{tabular}




\section{DISCUSSION}

In our study Group A streptococci (GAS) streptococcus pyogenes remains as the predominant species of streptococci causing majority of the infections. (42.42.\%) It has been isolated from clinical samples collected from cases of brain abscess, septic arthritis, cellulitis, pleural effusion and abscesses in different parts of the body. It is the most common isolate obtained from blood in cases of infective endocarditis. $(75 \%)$

Group B (Streptococci (GBS) otherwise known as streptococcus agalactiae is the second most common isolate in this study (18.18\%). It has been isolated from samples obtained from gluteal abscess, perianal abscess and leg ulcers.

Group D Streptococci (GDS) (enterococcus faecalis) obtained in this study was $(12.12 \%)$ was the isolates were obtained from cases of Infective endocarditis and abscesses.

Group F Streptococci (GFS) (Streptococci anginosus was first described in 1934 by Long and Bliss .Thy are minute haemolytic streptococci. Two isolates $(6.06 \%)$ obtained from abscess in this study.

Of the total number of 33 isolates by latex agglutination techniques, 7 isolates are nongroupable $(21.21 \%)$. The predominant isolates are group Astreptococci (42\%) common age group between $50-70 \mathrm{yrs}(40 \%)$ with male predominance (79\%).

Lancefield designations are often used to communicate medical microbiologists in the United States. Though there are many groups of streptococcus only five are known to commonly known to cause diseases in immune - competent humanbeings especially A, B and D. The two groups that lack the Lancefield carbohydrate antigen are streptococcus pneumoniae and viridans streptococci.

A number of important clinical and epidemiological studies have been carried out which have led to considerable improvement in the diagnosis, therapy and partly the prevention of these diseases. In spite of these advances, morbidity from streptococcal infections continuous to be relatively high. Streptococcal infections being among the most frequent bacterial infections of man and are most widely present in human beings as evidenced by a very high carrier rate as well as infection rate.

\section{CONCLUSION}

Streptococci can infect different parts of the body resulting in diversity of clinical entities. The most frequently encountered and the most important are the infections of the throat and skin especially with group A streptococci which has the unique ability to cause non-suppurative sequelae like rheumatic fever / rhematic heart disease and acute glomerulonephritis.Group A streptococci are widely distributed in the general population. Carrier rate in childhood varies from 5\% to $30 \%$. Streptococcal pharyngitis is one of the most common bacterial infections in children between the age group 3-5 yrs, peak rate occurs during the age span of 5-7 years. A second peak occurs during 12-13 years during change of school. Skin diseases like impetigo and pyoderma, are caused by different strains which are different from strains causing Respiratory tract infections. They never cause rheumatic fever through implicated in the production of Acute Glomerulonephritis after infection with nephritogenic strains. Wound infections caused by group A streptococci are primarily nosocomial in origin Rarely after respiratory tract infections but mostly after local sepsis it produces septicaemia mostly in the aged people Large no. of such infections are nosocomial. Group B streptococci has increasing association with neonatal sepsis, usually acquired from the carrier mothers, though nosocomial spread is also found. It also causes urinary tract infection in compromised hosts.

Group $\mathrm{C}$ and $\mathrm{G}$ can occasionally produce pharyngitis similar in clinical picture with group A streptococci but incapable of evoking nonsuppurative complications.

Lancefield grouping was done previously for definite classification and epidemiological studies. 
The conventional method was a tedious procedure time consuming and requiring lot of materials and manpower also. Streptococci are grown in ToddHewitt broth and the extraction of the specific Carbohydrate antigen done with any one of the methods such as Lancefield's acid extraction method using Hydrochloric acid, Fuller's method using form amide and Maxted's method using an enzyme produced by Streptomyces albus. The extract and the specific antisera are allowed to react in capillary tubes. Precipitation occurs within five minutes.

Besides capillary previpitation, grouping was also done by agar gel precipitation also.

Latex agglutination is a very simple, rapid test and easy to perform in any of the clinical laboratories with minimum basic facilities. Grouping of the isolate may be determined within minutes. The clinician will be able to select the antibiotic of choice for the group so that the treatment can be started at the earliest to prevent complications. So the latex agglutinations test for Lancefield grouping of beta-haemolytic streptococci may be recommended as a routine test to be performed in clinical laboratories getting large number of samples sent by clinicians suspecting streptococcal infections.

\section{REFERENCES}

1. Harvey, C.L. Mellmurray, M.B. Eur.J. Clinical Microbiology 3:6:526 (1984)

2. Holt et al (1004) Bergey's manual of Determinative Bacteriology ( $9^{\text {th }}$ Edition) Lippincott, Williams \& Wilkins. ISBN 0683-00603-7.

3. Kohler W. (June 2007). The present state of species within the genera streptococcus and Enterococcus. International Journal of Medical Microbiology 297 (3):133-50.

4. Cohen - Poradosu r, kasper DL. (2007). Group A streptococcus epidemiology and vaccine implications. Clin. Infect. Disease 45 (7):863-5.
5. Streptococcal infections (Invasive Group A strep) New York City Department of Health and Mental Hygiene. Nov. 2012.

6. Isenberg, Henry D. (1992) clinical microbiology procedures handbook. Washington, D.C: American Society of Microbiology.

7. Damask LJ, Montoya O, Axeford JL. Rapid slide agglutination test for Lancefied grouping of streptococcal. Arch Pathol Lab Med 1979 Aug : 103 (9) 456-8.

8. Kaufhold A and Ferrieri P. The Microbiolgic aspects, including and eterococcal infections. Infect Dis Clin North Am. 1993 June 7(2): 235-56.

9. Facklam R. What happened to the streptococci overview of taxonomic and nomendature changes. ClinMircobiol Rev. 2002 Oct.: 15(4):613-30.

10. Slifkin M, E, Engwall C, Puchet GR. Direct plate serological grouping of beta haemolytic streptococci from primary isolation plates with the phadebact streptococcus test. J. ClinMicrobiol. 1978 April: 7(4):356-60. 